PROCEEDINGS OF THE AMERICAN MATHEMATICAL SOCIETY

Volume 124, Number 7, July 1996

\title{
RESONANCE AND NON-RESONANCE IN A PROBLEM OF BOUNDEDNESS
}

\author{
RAFAEL ORTEGA AND ANTONIO TINEO
}

(Communicated by Hal L. Smith)

\begin{abstract}
This paper studies the existence of bounded solutions of a forced non-linear differential equation of arbitrary order. Necessary and sufficient conditions for the existence of such solutions are obtained. These results are inspired by classical results on the periodic problem, both in the resonant and non-resonant cases.
\end{abstract}

\section{INTRODUCTION}

Consider the differential equation

$$
y^{(n)}+a_{n-1} y^{(n-1)}+\cdots+a_{1} y^{\prime}+a_{0} y+g(y)=p(t)
$$

where $a_{0}, a_{1}, \ldots, a_{n-1}$ are real constants, $g: \mathbb{R} \rightarrow \mathbb{R}$ is continuous and bounded and $p: \mathbb{R} \rightarrow \mathbb{R}$ is an arbitrary continuous function. In this paper we study the existence of bounded solutions; that means solutions such that $y$ and the derivatives $y^{\prime}, \ldots, y^{(n-1)}$ are bounded in the whole real line. This boundedness problem can be seen as a non-standard boundary value problem and the results obtained in this paper will be reminiscent of some classical theorems on the existence of periodic solutions.

To motivate our results we first review some known facts on the periodic problem. Consider the linear differential operator

$$
L[y]:=y^{(n)}+a_{n-1} y^{(n-1)}+\cdots+a_{1} y^{\prime}+a_{0} y
$$

and assume first that the only $T$-periodic solution of $L[y]=0$ is $y=0$ (nonresonance). In this case (1) has at least one $T$-periodic solution if $p$ is $T$-periodic. A more difficult situation appears when $L[y]=0$ has non-trivial $T$-periodic solution (resonance). The existence results now depend on the nature of the periodic solutions of $L[y]=0$. Assuming that the only $T$ - periodic solutions of $L[y]=0$ are constants and that $g$ satisfies

$$
g(-\infty)<g(\xi)<g(+\infty) \quad \forall \xi \in \mathbb{R},
$$

it is possible to give a necessary and sufficient condition for the solvability of the periodic problem for (1), namely,

$$
g(-\infty)<\bar{p}<g(+\infty)
$$

Received by the editors January 18, 1995.

1991 Mathematics Subject Classification. Primary 34B15, 34C11.

(C)1996 American Mathematical Society 
where $\bar{p}=\frac{1}{T} \int_{0}^{T} p(s) d s$ is the average of the $T$-periodic function $p$. These results can be seen in ([6], p. 205, [8], [10], p. 168). Let us now assume that $p$ is an arbitrary function (not necessarily bounded or periodic). We look for bounded solutions and distinguish two cases. If the only bounded solution of $L[y]=0$ is $y=0$, it will be proven that (1) has a bounded solution if and only if $p$ can be decomposed in the form $p=p^{*}+p^{* *}$ where $p^{*}$ has a bounded primitive and $p^{* *}$ is bounded. This can be seen as a non-resonance result. In the resonant case we study the simplest situation: the constants are the only bounded solutions of $L[y]=0$ and they are simple (the exact meaning of this assumption will be made precise later). Assuming that (2) holds, we obtain a necessary and sufficient condition for the boundedness problem in the line of [3]. The concept of average does not have a natural extension for arbitrary continuous functions, but we replace it by the upper and lower averages (introduced in [7]) in our generalization of (3).

The standard proofs of existence in the periodic problem use an appropriate functional setting together with degree theory. They do not seem to extend in a direct way to the bounded problem and we replace them by proofs based on the method of guiding functions developed in [3]. The same ideas were already employed in [4] when the linear operator was

$$
L[y]=y^{\prime \prime}+c y^{\prime} \quad(c>0) .
$$

The results in [4] for this particular case are similar to the present results, although the notion of generalized average was not employed in [4] and this fact made the statements of the theorems more complicated. We point out that the extension of the proofs of [4] to the general case treated here is not trivial. In [4] it was sufficient to find explicitly one guiding function. Here the proof of the resonance result will require the use of a couple guiding functions that are defined in terms of the theory of exponential dichotomies.

To end this introduction we mention several other works dealing with the boundeness problem from different perspectives: [1], [3], [5], [9], [10].

The paper is organized in the following way. The main theorems are stated in $\S 2$. Some preliminary results on linear equations are given in $\S 3$. Proofs are given in $\S 4$.

\section{FUnCtion SPACES, GENERALIZED AVERAGES AND MAIN THEOREMS}

Let $C$ be the space of continuous functions $p: \mathbb{R} \rightarrow \mathbb{R}$ and consider the subspaces

$$
\begin{gathered}
C_{0}=\left\{p \in C: \sup _{t \in \mathbb{R}}\left|\int_{0}^{t} p(s) d s\right|<\infty\right\}, \\
B C=\left\{p \in C: \sup _{t \in \mathbb{R}}|p(t)|<\infty\right\}, \\
C_{0}+B C=\left\{p=p^{*}+p^{* *}: p^{*} \in C_{0}, p^{* *} \in B C\right\} .
\end{gathered}
$$

A solution $y$ of (1) is said to be bounded if $y, y^{\prime}, \ldots, y^{(n-1)} \in B C$. The existence of a bounded solution $y$ of (1) implies that the function $p$ can be split in the form $p=p^{*}+p^{* *}$ with $p^{*}=y^{(n)}, p^{* *}=a_{n-1} y^{(n-1)}+\cdots+a_{1} y^{\prime}+a_{0} y+g(y)$. This shows that a necessary condition for the existence of a bounded solution of (1) is

$$
p \in C_{0}+B C \text {. }
$$


In what follows we use the notation:

$$
\begin{gathered}
\varphi(\lambda)=\lambda^{n}+a_{n-1} \lambda^{n-1}+\cdots+a_{1} \lambda+a_{0} \quad(\text { characteristic polynomial of } L), \\
\underline{g}(+\infty)=\liminf _{\xi \rightarrow+\infty} g(\xi), \quad \bar{g}(-\infty)=\limsup _{\xi \rightarrow-\infty} g(\xi)
\end{gathered}
$$

(when the limits exist they are just denoted by $g( \pm \infty)$ ).

Theorem 1 (Non-resonance). Assume that $\varphi(\lambda)$ has no roots on the imaginary axis, $g \in B C$ and $p \in C$. Then (1) has at least one bounded solution if and only if

$$
p \in C_{0}+B C \text {. }
$$

To state the result in the case of resonance we follow [7] and define the notion of upper and lower average. Given $p \in C_{0}+B C$,

$$
\begin{aligned}
& A_{U}(p):=\lim _{r \rightarrow+\infty} \sup _{t-s \geq r} \frac{1}{t-s} \int_{s}^{t} p(z) d z, \\
& A_{L}(p):=\lim _{r \rightarrow+\infty} \inf _{t-s \geq r} \frac{1}{t-s} \int_{s}^{t} p(z) d z .
\end{aligned}
$$

It is easy to verify that

$$
-\infty<A_{L}(p) \leq A_{U}(p)<+\infty .
$$

Moreover, if $p$ is periodic the identity $A_{L}(p)=A_{U}(p)=\bar{p}$ holds and the concept of average is recovered.

Theorem 2 (Resonance). Assume that $\lambda=0$ is a simple root of $\varphi(\lambda)$ and there are no other roots on the imaginary axis. In addition, $g \in B C$ and $p \in C$. Then a sufficient condition for the existence of a bounded solution of (1) is

$$
p \in C_{0}+B C, \quad \bar{g}(-\infty)<A_{L}(p) \leq A_{U}(p)<\underline{g}(+\infty) .
$$

Moreover, if $g$ satisfies (2), the condition (4) is necessary and sufficient.

As mentioned in the introduction, this theorem extends results in [4]. The equation considered in [4] was

$$
y^{\prime \prime}+c y^{\prime}+g(y)=p(t) \quad(c>0),
$$

and it was proved that if $g \in B C$ and satisfies (2) there exists a bounded solution if and only if

$$
p=p^{*}+p^{* *} \text { with } p^{*} \in C_{0}, g(-\infty)<\inf p^{* *} \leq \sup p^{* *}<g(+\infty) .
$$

The conditions of Theorem 2 hold in this case and the next auxiliary result shows the connection between (4) and (5).

Lemma 3. Let $p \in C_{0}+B C$ be a given function and $\alpha, \beta \in \mathbb{R}, \alpha<\beta$. Then the following statements are equivalent:

(i) $\alpha<A_{L}(p) \leq A_{U}(p)<\beta$.

(ii) There exists a decomposition $p=p^{*}+p^{* *}$ with $p^{*} \in C_{0}, p^{* *} \in B C, \alpha<$ $\inf p^{* *} \leq \sup p^{* *}<\beta$.

Proof. (ii) $\Rightarrow$ (i) The definitions of upper and lower averages show that

$$
\inf p^{* *} \leq A_{L}\left(p^{* *}\right)=A_{L}(p) \leq A_{U}(p)=A_{U}\left(p^{* *}\right) \leq \sup p^{* *}
$$

and (i) follows. 
(i) $\Rightarrow$ (ii) It is similar to the proof of Lemma 2.2 in [4]. Let $P(t)=\int_{0}^{t} p(s) d s$ be a primitive of $p$. We first prove the existence of $a, b>0$ such that

$$
\left|P\left(t_{1}\right)-P\left(t_{2}\right)\right| \leq a+b\left|t_{1}-t_{2}\right| \quad \forall t_{1}, t_{2} \in \mathbb{R} \text {. }
$$

(Let $p=p_{1}+p_{2}$ be a decomposition of $p$ with $p_{1} \in C_{0}, p_{2} \in B C$. Then (6) holds with $a=2\left\|P_{1}\right\|_{\infty}, b=\left\|p_{2}\right\|_{\infty}$ and $P_{1}$ the primitive of $p_{1}$ with $P_{1}(0)=0$.) Now let $T$ and $\varepsilon$ be positive constants such that

$$
\alpha+\varepsilon<\frac{1}{T} \int_{t}^{t+T} p(s) d s<\beta-\varepsilon \quad \forall t \in \mathbb{R} .
$$

This is possible because (i) holds. Define

$$
p^{* *}(t)=\frac{1}{T} \int_{t}^{t+T} p(s) d s, \quad p^{*}=p-p^{* *} .
$$

By construction $p^{* *}$ satisfies all the requirements of (ii) and it remains to prove that $p^{*}$ belongs to $C_{0}$. Define $\Psi(t)=\int_{0}^{t} P(s) d s$; then

$$
P^{*}(t)=P(t)-\frac{1}{T}[\Psi(t+T)-\Psi(t)]
$$

is a primitive of $p^{*}$. From the mean value theorem we deduce that $P^{*}(t)=P(t)-$ $P(\xi)$ with $\xi \in[t, t+T]$. Thus $\left\|P^{*}\right\|_{\infty} \leq a+b T$ due to (6).

\section{Remarks on the linear EQUation}

We are now interested in the existence of bounded solutions of the nonhomogeneous linear equation

$$
L[y]=p(t)
$$

where $p \in C$. This question has been studied in [2] assuming that $p$ is bounded. We adapt the result of [2] to our context.

Lemma 4. Let $p \in C$ be given and assume that $\lambda=0$ is a simple root of the characteristic polynomial $\varphi(\lambda)$ and there are no other roots on the imaginary axis. Then (7) has a bounded solution if and only if $p \in C_{0}$.

Proof. The case $n=1$ is trivial. We assume $n \geq 2$. Let $y$ be a bounded solution of (7). Integrating

$$
P(t)=y^{(n-1)}(t)-y^{(n-1)}(0)+\cdots+a_{1}(y(t)-y(0))
$$

and $P$ is bounded.

Conversely, consider the equation

$$
y^{(n-1)}+a_{n-1} y^{(n-2)}+\cdots+a_{1} y=P(t) .
$$

Since this equation has an exponential dichotomy and we are assuming that $P$ is bounded, we can apply ([2], chapter 8$)$ to deduce the existence of a bounded solution. This function is also a bounded solution of the original equation (7).

Lemma 5. Let $p \in C$ be given and assume that $\varphi(\lambda)$ has no roots on the imaginary axis. Then (7) has a bounded solution if and only if $p \in C_{0}+B C$. 
Proof. The necessity of the condition $p \in C_{0}+B C$ was already discussed at the beginning of $\S 2$. To prove that it is also sufficient we assume that $p$ has the decomposition $p=p^{*}+p^{* *}, p^{*} \in C_{0}, p^{* *} \in B C$. Let $\mathcal{L}$ be a differential operator (different from $L$ ) of order $n$ and such that the conditions of Lemma 4 hold for $\mathcal{L}$. The equation $\mathcal{L}[y]=p^{*}$ has a bounded solution that we denote by $\varphi$. The change of variables $y=z+\varphi(t)$ transforms (7) into

$$
L[z]=\tilde{p}(t)
$$

with $\tilde{p}=p^{* *}+\mathcal{L}[\varphi]-L[\varphi]$. Since $\tilde{p}$ is bounded, the results of [2] imply the existence of a bounded solution $z$ of (8). The function $y=z+\varphi$ is a bounded solution of $(7)$.

\section{GUiding FUnCtions AND PROOFS}

We shall prove Theorems 1 and 2 using the ideas in [3] on the existence of bounded solutions of the general differential equation

$$
x^{\prime}=F(t, x)
$$

where $F: \mathbb{R} \times \mathbb{R}^{N} \rightarrow \mathbb{R}^{N}$ is continuous and locally Lipschitz continuous in $x$. A function $V \in C^{1}\left(\mathbb{R}^{N}\right)$ is a guiding function of (9) if there exists $R>0$ such that

$$
(\nabla V(x), F(t, x))>0 \quad \text { if }|x| \geq R, t \in \mathbb{R} .
$$

The index of $V$, ind $V$, is the degree of the field $\nabla V$ on large balls of $\mathbb{R}^{N}$. By a homotopy argument it is easy to see that the index only depends on the equation and not on the particular choice of $V$. The functions $V_{1}, \ldots, V_{r} \in C^{1}\left(\mathbb{R}^{N}\right)$ form a complete set of guiding functions if each of them is a guiding function and

$$
\lim _{|x| \rightarrow \infty}\left\{\left|V_{1}(x)\right|+\cdots+\left|V_{r}(x)\right|\right\}=\infty .
$$

A guiding function $V$ is called regular if there exist a constant $\alpha_{0}>0$ and a function $W \in C^{1}\left(\mathbb{R}^{N}\right)$ with $\lim _{|x| \rightarrow \infty}|W(x)|=\infty$ such that

$$
\begin{gathered}
(\nabla V(x), F(t, x))>\alpha_{0}|\nabla V(x)||F(t, x)| \quad \text { if }|x| \geq R, t \in \mathbb{R}, \\
|\nabla W(x)|<|\nabla V(x)| \quad \text { if }|x| \geq R .
\end{gathered}
$$

Proposition 6. In the previous notation assume that (9) has a complete set of guiding functions or a regular guiding function. In addition, the index of the guiding functions is non-zero. Then, there exists a solution $X=\left(x_{1}, \ldots, x_{N}\right)^{*}$ of $(9)$ with $x_{i} \in B C, 1 \leq i \leq N$. Moreover, the bounds on $X$ only depend on the guiding functions and $R$ but not on (9).

The proof is contained in [3].

Proof of Theorem 1. In order to apply Proposition 6 we assume that $g$ is locally Lipschitz-continuous. As in [4], the general case is treated using a regularization of $g$ and a passage to the limit. Assume that $p \in C_{0}+B C$ and let $\varphi$ be a bounded solution of $L[\varphi]=p(t)$. The existence of $\varphi$ is guaranteed by Lemma 5. The change of variables $y=z+\varphi(t)$ transforms (1) into

$$
L[z]+g(z+\varphi(t))=0
$$

and the transformation $x_{1}=z, \ldots, x_{n}=z^{(n-1)}$ leads to the system

$$
x^{\prime}=A x-g\left(x_{1}+\varphi(t)\right) e_{n}
$$


where

$$
A=\left(\begin{array}{rrrrr}
0 & 1 & 0 & \cdots & 0 \\
0 & 0 & 1 & \cdots & 0 \\
\vdots & \vdots & \vdots & \cdots & \vdots \\
-a_{0} & -a_{1} & -a_{2} & \cdots & -a_{n-1}
\end{array}\right), e_{n}=(0, \ldots, 1)^{*} .
$$

Since $A$ has no eigenvalues on the imaginary axis, it follows from ([2], chapter 7) that there exists a symmetric non-singular matrix $H$ such that

$$
H A+A^{*} H \leq-I_{n}
$$

(here $\leq$ refers to the standard order in the space of symmetric matrices). A computation shows that $V(x)=-(H x, x)$ is a regular guiding function, where $W=\lambda I$, $0<\lambda<\left\|H^{-1}\right\|^{-1}$. Since $H$ is non-singular, indV $\neq 0$ and Proposition 6 implies that the system (13) has a bounded solution. Since $\varphi$ is bounded together with $\varphi^{\prime}, \ldots, \varphi^{(n-1)}$, the same holds for (1).

Proof of Theorem 2. Necessary condition. If (2) holds and (1) has a bounded solution, $p$ has a decomposition of the kind $p=p^{*}+p^{* *}$ where $p^{* *}=g(y)$. Then $p^{*} \in C_{0}, g(-\infty)<\inf p^{* *} \leq \sup p^{* *}<g(+\infty)$ and (4) holds due to Lemma 3 .

Sufficient condition. By adding or substracting a constant we can modify $p$, and thus we can assume that

$$
\bar{g}(-\infty)<0<\underline{g}(+\infty)
$$

Also, as in the previous theorem, we assume that $g$ is locally Lipschitz-continuous. From (4) and Lemma 3 we find a decomposition $p=p^{*}+p^{* *}$ with $p^{*} \in C_{0}$ and

$$
\bar{g}(-\infty)<\inf p^{* *} \leq \sup p^{* *}<\underline{g}(+\infty) .
$$

It follows from Lemma 4 that the linear equation $L[y]=p^{*}(t)$ has a bounded solution $\varphi$. The change of variables $y=z+\varphi(t)$ transforms (1) into

$$
L[z]+g(z+\varphi(t))=p^{* *}(t)
$$

which is equivalent to

$$
x^{\prime}=A x-\left[g\left(x_{1}+\varphi(t)\right)-p^{* *}(t)\right] e_{n}
$$

(the same notation as in the proof of Theorem 1 is used). The condition (14) implies the existence of positive constants $\mu, \vartheta$ such that

$$
\xi\left[g(\xi+\varphi(t))-p^{* *}(t)\right] \geq \mu|\xi|-\vartheta \quad \forall(t, \xi) \in \mathbb{R}^{2} .
$$

We now apply Proposition 6 to (15). For $n=1$ the function $V(x)=-x^{2}$ is a regular guiding function. This can be proved using (16). For $n \geq 2$ we shall find a complete set of guiding functions $V_{1}, V_{2}$. To construct them we introduce the notation

$$
\begin{aligned}
& x=\left(x_{1}, \widehat{x}\right), \quad x_{1} \in \mathbb{R}, \quad \widehat{x} \in \mathbb{R}^{n-1}, \\
& \widehat{a}=\left(a_{2}, \ldots, a_{n-1}, 1\right)^{*}, \quad \widehat{e}=(0, \ldots, 0,1)^{*} \in \mathbb{R}^{n-1}, \\
& A=\left(\begin{array}{r|rrrr}
0 & 1 & 0 & \ldots & 0 \\
\hline \multirow{2}{*}{0} & & \multirow{A}{*}{} & \\
& & &
\end{array}\right), \widehat{A}=\left(\begin{array}{rrrrr}
0 & 1 & 0 & \ldots & 0 \\
0 & 0 & 1 & \cdots & 0 \\
\vdots & \vdots & \vdots & \ldots & \vdots \\
-a_{1} & -a_{2} & -a_{3} & \cdots & -a_{n-1}
\end{array}\right) \text {. }
\end{aligned}
$$


Since 0 is a simple root of $\varphi(\lambda)$, we know that $a_{1} \neq 0$. Also, from the assumptions on $\varphi(\lambda)$, we deduce that $\widehat{A}$ has no eigenvalues on the imaginary axis. As in the proof of Theorem 1 we find a non-singular symmetric matrix $\widehat{H}$ such that

$$
\widehat{H} \widehat{A}+\widehat{A}^{*} \widehat{H} \leq-I_{n-1} \text {. }
$$

Define $V_{1}(x)=-(\widehat{H} \widehat{x}, \widehat{x})-a_{1}\left[(\widehat{a}, \widehat{x})+a_{1} x_{1}\right]^{2}, \quad x \in \mathbb{R}^{n}$. Then

$$
\begin{aligned}
\dot{V}_{1}(x)= & -\left(\left(\widehat{H}+\widehat{A}+\widehat{A}^{*}+\widehat{H}\right) \widehat{x}, \widehat{x}\right)+2\left(g\left(x_{1}+\varphi(t)\right)-p^{* *}(t)\right)(\widehat{H} \widehat{e}, \widehat{x}) \\
& +2 a_{1}\left\{(\widehat{a}, \widehat{x})+a_{1} x_{1}\right\}\left(g\left(x_{1}+\varphi(t)\right)-p^{* *}(t)\right) \\
\geq & |\widehat{x}|^{2}-2\left(\|g\|_{\infty}+\left\|p^{* *}\right\|_{\infty}\right)\left[|\widehat{H} \widehat{e}|+2\left|a_{1} \| \widehat{a}\right|\right]|\widehat{x}| \\
& +2 a_{1}^{2} x_{1}\left[g\left(x_{1}+\varphi(t)\right)-p^{* *}(t)\right]
\end{aligned}
$$

and, in view of (16), there exist positive constants $\alpha, \beta, \gamma$ such that

$$
\dot{V}_{1}(x) \geq|\widehat{x}|^{2}-\alpha|\widehat{x}|+\beta\left|x_{1}\right|-\gamma \text { for each } x=\left(x_{1}, \widehat{x}\right) \in \mathbb{R}^{n} .
$$

Thus $V_{1}$ is a guiding function. We notice that, as a quadratic form, $V_{1}$ is nondegenerate. This happens with any quadratic form that is at the same time a guiding function. Consequently, $i n d V_{1} \neq 0$ and in fact $\left|i n d V_{1}\right|=1$. When $V_{1}$ is definite (positive or negative) the proof is complete since

$$
\lim _{|x| \rightarrow \infty}\left|V_{1}(x)\right|=\infty .
$$

However, if $V_{1}$ is not definite we need a second guiding function to construct a complete system. Notice that, in constrast to Theorem $1, V_{1}$ is not necessarily regular. Define $V_{2}(x)=V_{1}(x)+\delta\left[\frac{1}{2}|\widehat{x}|^{2}+G\left(x_{1}\right)\right]$ where $G(\xi)=\int_{0}^{\xi} g(\sigma) d \sigma$ and $\delta$ is a positive and small constant. Since we assumed $\bar{g}(-\infty)<0<\underline{g}(+\infty)$,

$$
\lim _{|\xi| \rightarrow \infty} G(\xi)=+\infty
$$

and $\left|V_{1}(x)\right|+\left|V_{2}(x)\right| \geq \delta\left[\frac{1}{2}|\widehat{x}|^{2}+G\left(x_{1}\right)\right] \longrightarrow+\infty$ as $|x| \longrightarrow \infty$. It remains to prove that $V_{2}$ is a guiding function. In fact

$$
\begin{aligned}
\dot{V}_{2}(x) & =\dot{V}_{1}(x)+\delta\left[(\widehat{x}, \widehat{A} \widehat{x})-\left[g\left(x_{1}+\varphi(t)\right)-p^{* *}(t)\right] x_{n}+g\left(x_{1}\right) x_{2}\right] \\
& \geq \dot{V}_{1}(x)-\delta\left[\|\widehat{A}\||\widehat{x}|^{2}+2\left(\|g\|_{\infty}+\left\|p^{* *}\right\|_{\infty}\right)|\widehat{x}|\right],
\end{aligned}
$$

and this estimate together with (17) shows that $V_{2}$ is a guiding function if $\delta$ is sufficiently small.

\section{REFERENCES}

[1] S. Ahmad, A nonstandard resonance problem for ordinary differential equations, Trans. Am. Math. Soc, 323 (1991), 857-875. MR 91e:34046

[2] W. A. Coppel, Dichotomies in Stability Theory, Lectures Notes in Math 629, Springer-Verlag, Berlin 1978. MR 58:1332

[3] M. A. Krasnoselskii, P. P. Zabreiko, Geometrical Methods of Nonlinear Analysis, SpringerVerlag, Berlin 1984. MR 85b:47057

[4] R. Ortega, A boundedness result of Landesman - Lazer type, Differential and Integral Equations, 8 (1995), 729-734. CMP 95:05

[5] G. Reuter, Boundedness theorems for nonlinear differential equations of the second order (II), J. London Math. Soc., 27 (1952), 48-58. MR 13:844b 
[6] N. Rouche, J. Mawhin, Equations Differentielles Ordinaires, Masson, Paris $1973 . \quad$ MR $\mathbf{5 8 : 1 3 1 8 b}$

[7] A. Tineo, An iterative scheme for the N-competing species problem, J. Diff. Eq. 116 (1995), $1-15$.

[8] J. R. Ward, Asymptotic conditions for periodic solutions of ordinary differential equations, Proc Amer Math Soc, 81 (1981), 415-420. MR 82a:34057

[9] J. R. Ward, A topological method for bounded solutions of nonautonomous ordinary differential equations, Trans. Am. Math. Soc.,333 (1992), 709-720. MR 93b:34046

[10] T. Yoshizawa, Stability Theory and the Existence of Periodic Solutions and Almost Periodic Solutions, Springer-Verlag, New York 1975. MR 57:6673

Departamento de Matemática Aplicada, Universidad de Granada, 18071 Granada, SPAIN

E-mail address: rortega@goliat.ugr.es

Departamento de Matemáticas, Facultad de Ciencias, Universidad de los Andes, 5101MÉRIDA, VENEZUELA

E-mail address: atineo@ciens.ula.ve 